\title{
Adenosine deaminase (ADA) isoenzymes ADA1 and ADA2 in biological fluids
}

To the Editor:

We read with great interest the editorial by GAKIS [1] about the extreme importance of the adenosine deaminase (ADA) isoenzymes ADA1 and ADA2 on the homeostasis of 2' deoxyadenosine and adenosine, especially when monocytes and macrophages are infected by intracellular microorganisms. Serum ADA activity is increased in various conditions such as liver disease, tuberculosis, typhoid, infective mononucleosis and certain malignancies, especially those of haematopoietic origin. The origin of serum ADA and the mechanisms by which serum activities are increased have not been fully elucidated.

Whatever the biological role of ADA1 and ADA2, it has been demonstrated that the presence (low or high) of these isoenzymes in biological fluids has diagnostic relevance. GAKIS [1] reviewed the known data on ADA activity in serum, pleural, peritoneal and pericardiac effusions, and cerebrospinal fluid (CSF) of many illnesses. To estimate the precise quantity of ADA1 and ADA2 in a specimen, one should separate the two isoenzymes. This was usually highly complicated and impractical, and some authors $[1,2]$ have introduced the 2 'deoxyadenosine/adenosine deaminase ratio. The ratio between the rate of deamination of 2'deoxyadenosine and adenosine, performed independently in two separate test tubes, will have different values according to the relative percentages of the two isoenzymes on the specimen. Estimated data are calculated using the affinity of ADA2 for the two substrates.

However, we feel that this is not the most practical way to obtain a measurement of the relative percentage of ADA1 and ADA2 activity in the sample, especially when the readings of ADA activity can be made by an automatic analyser system with reagent kits. In our current work, we incorporated the erythro-9-(2-hydroxy3-nonyl) adenine (EHNA), which specifically and completely inhibited only the ADA1 isoenzymes fraction [3]. Whatever the method used to measure ADA activity, an exact relationship between the isoenzymes is readily obtained by measuring total ADA and the ADA2 which is not inhibited by $100 \mathrm{mmol} \cdot \mathrm{L}^{-1}$ of added EHNA. In fact, this method is being increasingly used by acquired immune deficiency syndrome (AIDS) researchers since some authors have reported a higher serum ADA activity in human immunodeficiency virus (HIV)-1 positive patients [4]. By the method we propose, some authors have found that ADA2 isoenzyme activity is of considerable prognostic value in AIDS and adult T-cell leukaemia (ATL) cases $[5,6]$.

\section{References}

1. Gakis C. Adenosine deaminase (ADA) isoenzymes ADA1 and ADA2: diagnostic and biological role. Eur Respir J 1996; 632-633.

2. Valdés L, San José E, Alvarez D, Valle JM. Adenosine deaminase (ADA) isoenzyme analysis in pleural effusions: diagnostic role, and relevance to the origin of increased ADA in tuberculous pleurisy. Eur Respir $J$ 1996; 9: 747-751.

3. Ungerer JPJ, Oosthuizen HM, Bissbort SH, Vermaak WJH. Serum adenosine deaminase: isoenzymes and diagnostic application. Clin Chem 1992; 38: 1322-1326.

4. Valls V, Ena J, Roca V, Pérez-Oteyza C, Figueredo MA, Enriquez-de-Salamanca R. Significance of adenosine deaminase measurement in sera of patients with HIV-1 infection. AIDS 1990; 4: 365-366.

5. Yokoyama MM, Tsuboi I. Adenosine deaminase isoenzymes and HIV/HTLV-1 infections. J Natl Cancer Inst 1988; 80: 698.

6. Matsuda J, Tsukamoto M, Gohchi K, Saitoh N, Kawasugi K, Kinoshita T. Serum adenosine deaminase 2 and neopterin levels are increased in a majority of hemophiliacs irrespective of infection with human immunodeficiency virus type 1. Clin Infect Dis 1993; 16: 260-264.

\section{O.J. Cordero, F.J. Salgado, M. Nogueira.}

University of Santiago de Compostela, Dept of Biochemistry and Molecular Biology, Facultade de Bioloxía, 15706 Santiago de Compostela, Spain.

\section{REPLY}

\section{From the authors:}

Cordero et al. propose a method for measuring ADA1 and ADA2 in biological fluids as a more practical approach than that based on the substrate affinity of the two isoenzymes [1]. By using an automatic analyser system, they incorporated erythro-9-(2-hydroxy-3-nonyl) adenine (EHNA), "which specifically and completely inhibited only the ADA1 isoenzymes fraction". They

state that "whatever the method used to measure ADA activity, an exact relationship between the isoenzymes is readily obtained by measuring total ADA and the ADA2 which is not inhibited by $100 \mu \mathrm{mol} \cdot \mathrm{L}^{-1}$ of added EHNA". A few papers are quoted to support their thesis.

I regret that I could not find a detailed description of the putative new method in the letter from Cordero et al. or in any of the quoted papers. In particular, I did not find any information on the amount of adenosine and 2 'deoxyadenosine in the mixture of enzymatic reaction. 
It may be that EHNA is useful to determine ADA2 in biological fluids, but with the presented data, I cannot evaluate the method. Furthermore, I could not reproduce the method because EHNA is not commercially available.

While commenting on their references, Cordero et al. also say: "... ADA2 isoenzyme activity is of considerable prognostic value in AIDS and adult T-cell leukaemia (ATL) cases". ADA2 activity in HIV-positive subjects is due to the presence of HIV inside monocytes and macrophages and has no diagnostic or prognostic significance $[1,2]$. One should also consider that ADA2 activity in HIV-positive subjects could be due to concomitant infections by intracellular parasites, which are exceedingly common in these patients. In patients with systematic tumours, it is ADA1 that has a clear diagnostic usefulness $[1,3,4]$. On the contrary, ADA2 increases in a few cases of lymphoproliferative diseases, but it does not allow a differential diagnosis versus infectious disease due to intracellular microorganisms where ADA2 is also increased $[1,2,4]$.

\section{References}

1. Gakis C, Naitana A, Ortu AR, Contu A, Bechere M. Adenosine deaminase activity in the diagnosis of infectious diseases. Infect Med 1994; 219-232.

2. Gakis C, Calia G, Naitana A, Pirino D, Serru G. Serum adenosine deaminase activity in HIV positive subjects. A hypothesis on the significance of ADA2. Panm Med 1989: 31: 107-113.

3. Gakis C, Piras MA, Romani G. Unusual biochemical pattern of serum adenosine deaminase activity in acute lymphoblastic leukemia. Haematologica 1982; 67: 199-206.

4. Gakis C, Calia G, Naitana A, Ortu AR, Contu A. Serum and pleural adenosine deaminase activity, correct interpretation of the findings. Chest 1991; 99: 1555-1556.

\section{Gakis}

Clinic of Infectious Diseases, University of Sassari, Sassari, Italy. 